\title{
Erratum to: Life Satisfaction as a Distinguishing Indicator of College Student Functioning: Further Validation of the Two-Continua Model of Mental Health
}

\author{
Tyler L. Renshaw $\cdot$ Alex S. Cohen
}

Published online: 29 November 2013

(C) Springer Science+Business Media Dordrecht 2013

\section{Erratum to: Soc Indic Res \\ DOI 10.1007/s11205-013-0342-7}

The corrected version of Table 4 is given below.

Table 4 Two-continua model of mental health classification schema

\begin{tabular}{lll}
\hline $\begin{array}{l}\text { Psychological } \\
\text { distress }\end{array}$ & Psychological well-being \\
\cline { 2 - 3 } & Languishing-to-mixed life satisfaction & Moderate-to-high life satisfaction \\
\hline Typical-range symptoms & $\begin{array}{c}\text { Asymptomatic yet discontent } \\
(18.7 \%, n=253)\end{array}$ & $\begin{array}{c}\text { Mentally healthy } \\
(61.4 \%, n=833)\end{array}$ \\
Clinical-range symptoms & Mentally unhealthy $(15.1 \%, n=205)$ & $\begin{array}{c}\text { Symptomatic yet content } \\
(4.8 \%, n=65)\end{array}$ \\
\hline
\end{tabular}

The online version of the original article can be found under doi:10.1007/s11205-013-0342-7.

T. L. Renshaw $(\bowtie) \cdot$ A. S. Cohen

Department of Psychology, Louisiana State University, 204 Audubon Hall, Baton Rouge,

LA 70803, USA

e-mail: trenshaw@1su.edu 